\title{
The Diversity and Dynamics of Indigenous Yeast Communities in Grape Must from Vineyards Employing Different Agronomic Practices and their Influence on Wine Fermentation
}

\author{
B. Bagheri, F.F. Bauer, M.E. Setati*
}

Institute for Wine Biotechnology, Stellenbosch University, P/Bag X1, Matieland, 7602, South Africa

Submitted for publication: November 2014

Accepted for publication: January 2015

Key words: Wine fermentation, yeast dynamics, non-Saccharomyces yeasts, fermentation kinetics

\begin{abstract}
The current study evaluated the diversity of yeast species in Cabernet Sauvignon grape must derived from three neighbouring vineyards from a similar terroir but on which significantly different management practices are employed. The fermentation kinetics and yeast population dynamics were monitored from the beginning to the end of spontaneous fermentation. The grape musts were characterised by distinct yeast populations comprising oxidative, weakly fermentative and strongly fermentative yeasts. Different combinations of dominant non-Saccharomyces yeasts were observed in each must, with significantly different assortments of dominant species, including Starmerella bacillaris (synonym Candida zemplinina), Lachancea thermotolerans, Hanseniaspora uvarum, Candida parapsilosis and Wickerhamomyces anomalus. None of these yeast consortia appeared to affect the growth of Saccharomyces cerevisiae or inhibit the overall progress of fermentation. However, the percentage of fermentative yeasts was positively correlated with the fermentation rate. Glucose and fructose consumption rates suggested active participation of both glucophilic and fructophilic yeasts from the onset of fermentation. The data highlight two parameters, viz. initial cell concentration and yeast community composition, as important fermentation drivers and open the possibility to predict fermentation behaviour based on the initial composition of the yeast community.
\end{abstract}

\section{INTRODUCTION}

Grapes harbour a wide variety of yeasts, many of which are intimately associated with the berry skin. The diversity and density of yeasts fluctuate throughout the berryripening stages (Prakitchaiwattana et al., 2004; Renouf et al., 2005; 2007; Barata et al., 2012a) and also vary between healthy and damaged berries. Typically, the yeast population associated with healthy grapes from the berry peppercorn stage until the time of harvest is characterised by a high incidence of basidiomycetous yeasts of the genera Cryptococcus, Rhodotorula and Rhodosporidium, as well as the ascomycetous yeast-like fungus Aureobasidium pullulans (Rementeria et al., 2003; Renouf et al., 2005; Barata et al., 2012a; Setati et al., 2012; Díaz et al., 2013). Fermentative yeasts, on the other hand, generally are numerically underrepresented on healthy grapes (Mortimer \& Polsinelli, 1999; Barata et al., 2012a), and studies have suggested that insects such as members of the genera Drosophila and Vespula act as the primary vectors of ascomycetous fermentative yeasts such as Saccharomyces spp., Saccharomycodes spp., Hanseniaspora uvarum, Candida spp., Pichia spp. and Zygoascus spp. (Mortimer \& Polsinelli, 1999; Barata et al., 2012b; Stefanini et al., 2012). Several factors, including agronomic practices, influence yeast diversity on grapes. In particular, farming practices such as the application of pesticides have been reported to affect yeast populations, including the diversity of fermentative yeast strains (CorderBueso et al., 2011; Tello et al., 2011; Tofalo et al., 2011; Setati et al., 2012). However, when considering the preponderance of non-fermentative yeasts in the vineyard microbiota, the impact of such practices on the fermentation microbiota has received less attention.

Today, most wine fermentations are inoculated with commercially produced active dried wine yeast strains. However, spontaneous fermentation, i.e. the fermentation by the microorganisms that are naturally present on grapes and in cellars, is again becoming more widespread, in particular because of the adoption of less interventionist practices that also are perceived as more environmentally friendly. Spontaneous fermentation, while presenting increased risks of off-flavour production and stuck fermentation, is furthermore believed to generate wines with more complex aroma and flavour, presumably due to the significant contribution of non-Saccharomyces yeasts (Tello et al., 2011).

*Corresponding author: E-mail address: setati@sun.ac.za [Fax: +27 21808 3771; Tel.: +27 218089203$]$

Acknowledgements: The authors would like to thank Winetech (grant IWBT-MG-11/01) and the National Research Foundation - Technology and Human Resources for Industry Programme (grant TP2011060600013) for funding. The opinions expressed and conclusions arrived at are those of the authors and are not necessarily to be attributed to the funding agencies 
The general pattern of the development of microbial populations during spontaneous wine fermentation is reasonably well established. Fresh grape juice typically contains between $10^{4}$ and $10^{6} \mathrm{CFU} / \mathrm{mL}$ yeast counts, which increases to $10^{8} \mathrm{CFU} / \mathrm{mL}$ during fermentation (Povhe Jemec et al., 2001; Combina et al., 2005; Di Maro et al., 2007). The kinetics of the main yeast species have been monitored using both culture-dependent approaches as well as cultureindependent methods, including FISH (Xufre et al., 2006), PCR-DGGE (Di Maro et al., 2007; Renouf et al., 2007) and qRT-PCR (Zott et al., 2008). These studies revealed rapid and significant increases in Saccharomyces cerevisiae strains and sometimes a dramatic decline in non-Saccharomyces species. The decline appears most likely to be associated with $\mathrm{SO}_{2}$ intolerance, sensitivity to anaerobic conditions, and intolerance of increasing levels of ethanol (Combina et al., 2004). However, since these traits are strain dependent, there have been reports of strains of $H$. uvarum, Issatchenkia orientalis, Pichia kluyveri, Starmerella bacillaris (synonym Candida zemplinina) (Duarte et al., 2012), Torulaspora delbrueckii and Lachancea thermotolerans that were able to persist until the middle and sometimes end of fermentation in significant numbers (Povhe Jemec et al., 2001; Sun et al., 2009; Bezerra-Bussoli et al., 2013; Milanovic et al., 2013; Tristezza et al., 2013), although $S$. cerevisiae remains the dominant yeast in these stages. The dynamics of $S$. cerevisiae strains are variable and may involve one or more strains, and the sequential substitution of different strains has also been reported (Sturm et al., 2006).

The kinetics and completion of fermentation are believed to be largely dependent on S. cerevisiae, and competition by a large number of non-Saccharomyces species is thought to be detrimental from a fermentation kinetic perspective. Here we evaluate the fermentation kinetics and population dynamics of three spontaneous fermentations of grape juices derived from three differently treated vineyards. The three vineyards were shown, using automated ribosomal intergenic spacer analysis (ARISA), to harbour distinct grape-associated yeast populations, although cultivation-dependent analyses could not show a clear distinction (Setati et al., 2012). The current study aimed to investigate whether the differences in the three vineyards were also evident in the yeast populations constituting the wine microbial consortium, and how differences in the initial yeast population composition and concentration would influence the fermentation kinetics. A better understanding of the initial diversity in grape must and the dynamics of the yeast population throughout spontaneous fermentation will help improve the management of such fermentations and potentially avoid sluggish fermentations and risks of spoilage.

\section{MATERIALS AND METHODS}

\section{Grape sampling and wine fermentations}

The grapes used in the current study were obtained from three vineyards that employ different management systems: biodynamic (33॰57’39.33" S, $18^{\circ} 45^{\prime} 13.46^{\prime \prime}$ E, elevation $183 \mathrm{~m})$, conventional (33 $57^{\prime} 41.50^{\prime \prime} \mathrm{S}, 18^{\circ} 45^{\prime} 11.87^{\prime \prime}$ $\mathrm{E}$, elevation $179 \mathrm{~m}$ ) and integrated pest management (335' $40.65^{\prime}$ ' S, $18^{\circ} 45^{\prime} 08.23^{\prime}$ 'E, elevation $184 \mathrm{~m}$ ). The conventional and biodynamic vineyards had the same
Cabernet Sauvignon rootstock (R101-14), while the integrated vineyard had rootstock R110-CS23A. The layout, location and chemical treatments of the vineyards were described in detail in a previous study (Setati et al., 2012). Samples were collected in two consecutive years (2012 and 2013) and used for microvinification. For each vineyard, $5 \mathrm{~kg}$ of Cabernet Sauvignon grapes were collected and transported to the laboratory in sterile "Ziploc ${ }^{\circledR}$ " bags. The grapes were aseptically hand-destemmed and crushed within $1 \mathrm{~h}$ of collection. The chemical composition of the must was analysed by Fourier transform infrared (FT-IR) spectroscopy using the GrapeScan 2000 instrument (FOSS Electric, Denmark). Fifty millilitre samples were collected from the fresh must and used for yeast isolation and enumeration. The remaining must was transferred into duplicate $2 \mathrm{~L}$ fermentation bottles and set up for natural fermentation at $25^{\circ} \mathrm{C}$ to allow sufficient growth of the non-Saccharomyces population without negatively affecting the development of the indigenous $S$. cerevisiae strains. Alcoholic fermentation was followed by measuring the loss of weight resulting from $\mathrm{CO}_{2}$ release. In addition, samples were withdrawn regularly for sugar analysis. The glucose and fructose concentrations were measured using the Enytec ${ }^{\mathrm{TM}}$ Fluid D-Glucose IdNo: 5140 and Enytec ${ }^{\mathrm{TM}}$ Fluid D-Fructose Id-No: 5120 (R-Biopharm AG, Germany) enzymatic kits on the Arena ${ }^{\mathrm{TM}}$ 20XT Photometric analyser (Thermo Electron, Oy, Finland). Yeast dynamics were evaluated after $12.5 \%, 30 \%, 50 \%$ and $70 \%$ sugar consumption.

\section{Yeast enumeration and isolation}

Samples were taken every two days for monitoring the yeast populations. Serial decimal dilutions were prepared in triplicate in $0.9 \%(\mathrm{w} / \mathrm{v}) \mathrm{NaCl}$ solution and spread on three different media: (i) Wallerstein nutrient (WLN) agar, supplemented with $34 \mu \mathrm{g} / \mathrm{mL}$ chloramphenicol and $200 \mu \mathrm{g} / \mathrm{mL}$ biphenyl to suppress bacteria and moulds, was used for total yeast enumeration, (ii) WLN agar with $34 \mu \mathrm{g} / \mathrm{mL}$ chloramphenicol, $200 \mu \mathrm{g} / \mathrm{mL}$ biphenyl and $1 \mu \mathrm{g} / \mathrm{mL}$ cycloheximide was used for the enumeration of the non-Saccharomyces (NS) population, and (iii) YPD agar supplemented with $12 \%$ ethanol, $25 \mathrm{mg} / \mathrm{L}$ kanamycin and $150 \mathrm{mg} / \mathrm{L} \mathrm{K}_{2} \mathrm{~S}_{2} \mathrm{O}_{5}$ was used for the enumeration of Saccharomyces species. Yeast enumeration and isolation were carried out on plates from the dilutions that contained between 30 and 300 colonies. Colonies with different characteristics (colour, texture, size, shape, margin) were isolated and purified through two rounds of streaking. A minimum of six representative colonies per colony morphology were streaked out from each plate where possible. The isolates obtained were stored in glycerol $20 \%$ $(\mathrm{v} / \mathrm{v})$ at $-80^{\circ} \mathrm{C}$.

\section{Yeast identification}

For the isolation of genomic DNA, yeast cells were grown in 5 $\mathrm{mL}$ YPD broth. DNA was extracted from a $1 \mathrm{~mL}$ sample using the rapid yeast DNA extraction method (Hoffman, 2003). The ITS1-5.8S rRNA-ITS2 region was amplified by PCR using the primer set ITS1 (5'-TCCGTAGGTGAACCTCGCG-3') and ITS4 (5'-TCCTCCGCTTTATTGATATGC-3') (EsteveZarzoso et al., 1999). PCR was performed in a final volume 
of $25 \mu \mathrm{L}$ containing $0.4 \mathrm{mM}$ dNTP mix, $0.25 \mu \mathrm{M}$ of each primer, $1 \mathrm{U}$ of Ex-Taq polymerase (TaKara), 1× buffer, $1 \mathrm{mM} \mathrm{MgCl}_{2}$ and $100 \mathrm{ng}$ template DNA. The PCR products were analysed on a $1 \%$ agarose gel and purified using the Zymoclean $^{\mathrm{TM}}$ Gel DNA recovery kit (Zymo Research Corporation, Irvine, CA, USA) following the manufacture's instruction. Restriction fragment polymorphisms of the ITS5.8S rRNA gene were analysed by digesting the PCR product with HaeIII, HinfI and $C f o \mathrm{I}$ in separate reactions as described by Esteve-Zarzoso et al. (1999). The isolates were grouped according to distinct restriction patterns, and previously sequenced species were digested with the same enzymes and used as references to identify the current isolates.

\section{Statistical analyses}

The relative abundance of species was calculated as a proportion of a particular species in the samples based on colony counts and frequency of isolation. Yeast species diversity was determined using the Shanon Weiner index and Simpson's index, as described by Cordero-Bueso et al. (2011). The Shanon index for diversity was calculated as follows:

$\mathrm{H}^{\prime}=-\sum^{s} p_{i} \log _{2}\left(p_{i}\right)$

where $s$ is the number of species and is the proportion of the species within the total population. Species dominance was determined on the basis of Simpson's index using the following equation:

$\mathrm{D}=\sum^{S}\left(p_{i}\right)^{2}$

\section{RESULTS}

\section{Isolation and identification of yeasts}

The chemical composition of the grape must from the three vineyards revealed differences in the sugar concentration, $\mathrm{pH}$ and tartaric acid concentrations. The total glucose and fructose concentrations ranged from $210 \mathrm{~g} / \mathrm{L}$ to $265 \mathrm{~g} / \mathrm{L}$, pH varied between 3.35 and 3.66, while tartaric acid concentrations were varied from 2.0 to $5.4 \mathrm{~g} / \mathrm{L}$ (Table 1).

The three farming systems displayed different yeast community composition in the initial musts. Since the evaluation was culture based, only yeast species that represented $>1 \%$ of the total yeast population are reported here, since all other species would likely not have any significant impacts on the system. In general, total species numbers were low, indicating the rapid die-off of many yeast species that were prominent on the grapes. The data show that, overall, the 2013 vintage displayed more diversity than the 2012 in all musts (Table 2). In total, eight, four and one yeast species were identified in the 2012 musts, whereas 12, 11 and nine species were identified in the 2013 musts obtained from the biodynamic, "conventional" and "integrated" vineyards respectively. During the 2012 vintage, Aureobasidium pullulans and Kazachstania aerobia were the two dominant yeasts in the biodynamic and conventional vineyards, whereas Hanseniaspora uvarum was the only yeast isolated in the integrated vineyard in 2012. In contrast, a more diverse yeast community, comprising A. pullulans, H. uvarum, Torulaspora delbrueckii, Wickerhamomyces anomalus (formerly Pichia anomala or Hansenula anomala), Rhodotorula spp., Rhodosporidium diobovatum, Candida azyma and Lachancea thermotolerans (formerly Kluyveromyces thermotolerans), was observed in the integrated vineyard in 2013 (Table 2). Saccharomyces cerevisiae could only be isolated from the initial must of the biodynamic vineyard in both vintages. Candida parapsilosis was also only isolated in the biodynamic vineyard. Other yeasts present included Cryptococcus spp., Rhodotorula spp. and Issatchenkia terricola. When the two vintages were taken together, the biodynamic vineyard exhibited the highest culturable diversity $\left(H^{\prime}=2.32\right)$ and the lowest dominance $(D=0.11)$, followed by the conventional vineyard $\left(H^{\prime}=2.23\right.$ and $D=0.17)$, while the integrated vineyard revealed lower diversity $\left(H^{\prime}=1.15\right)$ and the highest dominance $(D=0.37)$.

\section{General fermentation kinetics}

The current study evaluated spontaneous fermentation kinetics in grape must derived from biodynamic, integrated and conventional farming systems. Two vintages were analysed and similar fermentation trends were observed. The initial population of NS yeasts varied from $10^{4}$ to $10^{6}$ $\mathrm{CFU} / \mathrm{mL}$, depending on the vintage, with minor variations between the farming systems. Data from the 2013 vintage are presented to demonstrate the trends observed. The must from the biodynamic vineyard had an initial total yeast population of $2.3 \times 10^{6} \mathrm{CFU} / \mathrm{mL}$ and the fermentation proceeded rapidly, while fermentation of the musts from the conventional and integrated vineyards had an initial yeast load of $2.35 \times 10^{4}$ and $8.43 \times 10^{4} \mathrm{CFU} / \mathrm{mL}$ respectively. Both fermentations displayed a short lag phase, but took

TABLE 1

Chemical composition of grape musts prepared in 2012 and 2013.

\begin{tabular}{lllcccc}
\hline \multirow{2}{*}{ Parameter } & \multicolumn{2}{c}{ Biodynamic } & \multicolumn{2}{c}{ Conventional } & \multicolumn{2}{c}{ Integrated } \\
& $\mathbf{2 0 1 2}$ & $\mathbf{2 0 1 3}$ & $\mathbf{2 0 1 2}$ & $\mathbf{2 0 1 3}$ & $\mathbf{2 0 1 2}$ & $\mathbf{2 0 1 3}$ \\
\hline Glucose/fructose (g/L) & $239 \pm 0.20$ & $264 \pm 1.00$ & $210 \pm 0.40$ & $265 \pm 0.60$ & $249 \pm 0.30$ & $239 \pm 0.40$ \\
'Brix & $23.4 \pm 0.00$ & $25.4 \pm 0.09$ & $20.7 \pm 0.00$ & $25.6 \pm 0.02$ & $24.3 \pm 0.02$ & $23.4 \pm 0.02$ \\
Titratable acidity (g/L) & $3.23 \pm 0.01$ & $3.96 \pm 0.003$ & $3.85 \pm 0.02$ & $4.28 \pm 0.01$ & $2.37 \pm 0.04$ & $3.65 \pm 0.07$ \\
pH & $3.61 \pm 0.001$ & $3.50 \pm 0.013$ & $3.35 \pm 0.02$ & $3.49 \pm 0.02$ & $3.66 \pm 0.002$ & $3.55 \pm 0.02$ \\
Tartaric acid (g/L) & $3.10 \pm 0.05$ & $4.80 \pm 0.01$ & $2.30 \pm 0.09$ & $5.40 \pm 0.01$ & $3.20 \pm 0.03$ & $5.00 \pm 0.02$ \\
Malic acid (g/L) & $0.90 \pm 0.005$ & $0.70 \pm 0.17$ & $1.40 \pm 0.10$ & $0.30 \pm 0.03$ & nd & $0.55 \pm 0.02$ \\
Volatile acidity (g/L) & $0.4 \pm 0.001$ & $0.41 \pm 0.02$ & $0.31 \pm 0.02$ & $0.32 \pm 0.02$ & $0.32 \pm 0.01$ & $0.27 \pm 0.01$ \\
\hline
\end{tabular}

nd: not detected 
TABLE 2

Percentage yeast species distribution in grape must from biodynamic, conventional and integrated vineyard in two consecutive years.

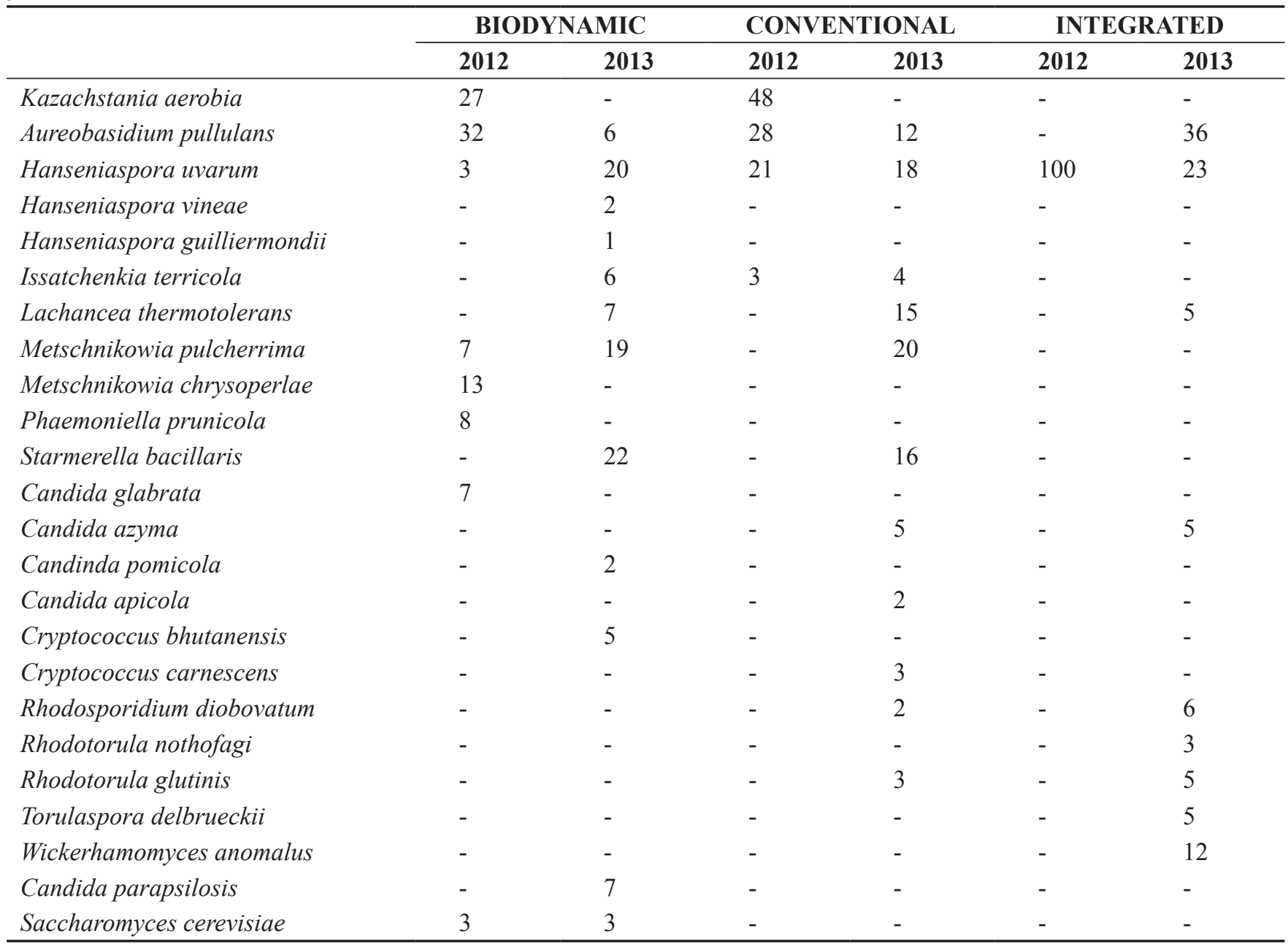

almost twice the amount of time to complete compared to the must from the biodynamic vineyard. The initial total yeast population in the must from the biodynamic vineyard increased from $10^{6}$ to $10^{8} \mathrm{CFU} / \mathrm{mL}$, after which it stabilised until the end of fermentation, with a slight decrease to $10^{7}$ CFU/mL (Fig. 1). The non-Saccharomyces yeast population displayed a notable increase from $10^{6}$ to $10^{8} \mathrm{CFU} / \mathrm{mL}$ after four days of fermentation, followed by a steady decline until it dropped below detection level after $90 \%$ of the sugar was consumed, whereas the Saccharomyces population increased rapidly to $10^{7} \mathrm{CFU} / \mathrm{mL}$ in the first two days of fermentation, reaching a maximum of $10^{8} \mathrm{CFU} / \mathrm{mL}$ and remaining stable until the end of fermentation. Similar trends were observed in the fermentation of the must from the conventional and integrated vineyards, even though the initial yeast population levels were lower and the fermentation took longer. In these two fermentations, the Saccharomyces population could only be detected two days after the onset of fermentation.

\section{Yeast dynamics throughout fermentation}

The oxidative yeasts, including A. pullulans, Rhodotorula spp., R. diobovatum and Cryptococcus spp., which accounted for 5,8 and $14 \%$ of the total initial yeast population in the must from the biodynamic, conventional and integrated vineyard respectively, dropped below detection when fermentation commenced (Fig. 2). The must from the biodynamic vineyard displayed 12 different species at the onset of fermentation; however, after $12.5 \%$ of the sugar had been consumed the number decreased to five species, comprising Candida parapsilosis (32\%), S. bacillaris (19.9\%), H. uvarum (25.4\%), M. pulcherrima (2.5\%) and S. cerevisiae (20.2\%). Similarly, in the must from the conventional vineyard the yeast diversity declined from 11 species to six species, viz. H. uvarum (30.4\%), L. thermotolerans (22.7\%), S. bacillaris (18\%), M. pulcherrima (12.5\%), C. azyma (7.1\%) and $S$. cerevisiae $(9.3 \%)$, in the first five days of fermentation. Nine different species were found in the must from the integrated vineyard. The number of species declined to five after $12.5 \%$ of the sugar had been consumed. H. uvarum $(66 \%)$ was the most dominant species, while $W$. anomalus (14\%), T. delbrueckii (8.9\%), C. azyma (4.6\%) and S. cerevisiae $(6.5 \%)$ were present at lower percentages. H. uvarum, which was present in all the fermentations, persisted until the middle of fermentation. In contrast, L. thermotolerans only persisted in the must from the conventional vineyard, where its initial level was higher ( $15 \%$ of the total population). This yeast increased up to $2 \times 10^{6} \mathrm{CFU} / \mathrm{mL}$ and then declined to $3 \times$ $10^{3} \mathrm{CFU} / \mathrm{mL}$ by late fermentation. S. bacillaris was detected 
in the must from the biodynamic and conventional vineyard and displayed similar dynamics in both fermentations. The cell concentration started with an increase up to $2.5 \times 10^{6}$ $\mathrm{CFU} / \mathrm{mL}$, followed by a decline during the tumultuous phase of fermentation. C. azyma, present in the conventional and integrated must, maintained the same concentration in the early stages of fermentation and then declined after $12.5 \%$ (w/v) of the sugar had been consumed (Fig. 2). The must from the biodynamic vineyard displayed a high incidence of C. parapsilosis, and this yeast remained dominant throughout fermentation, while $W$. anomalus was dominant in the integrated must, second to H. uvarum. In contrast, T. delbrueckii, which was only found in the integrated must, did not show any increase. In all the fermentations,

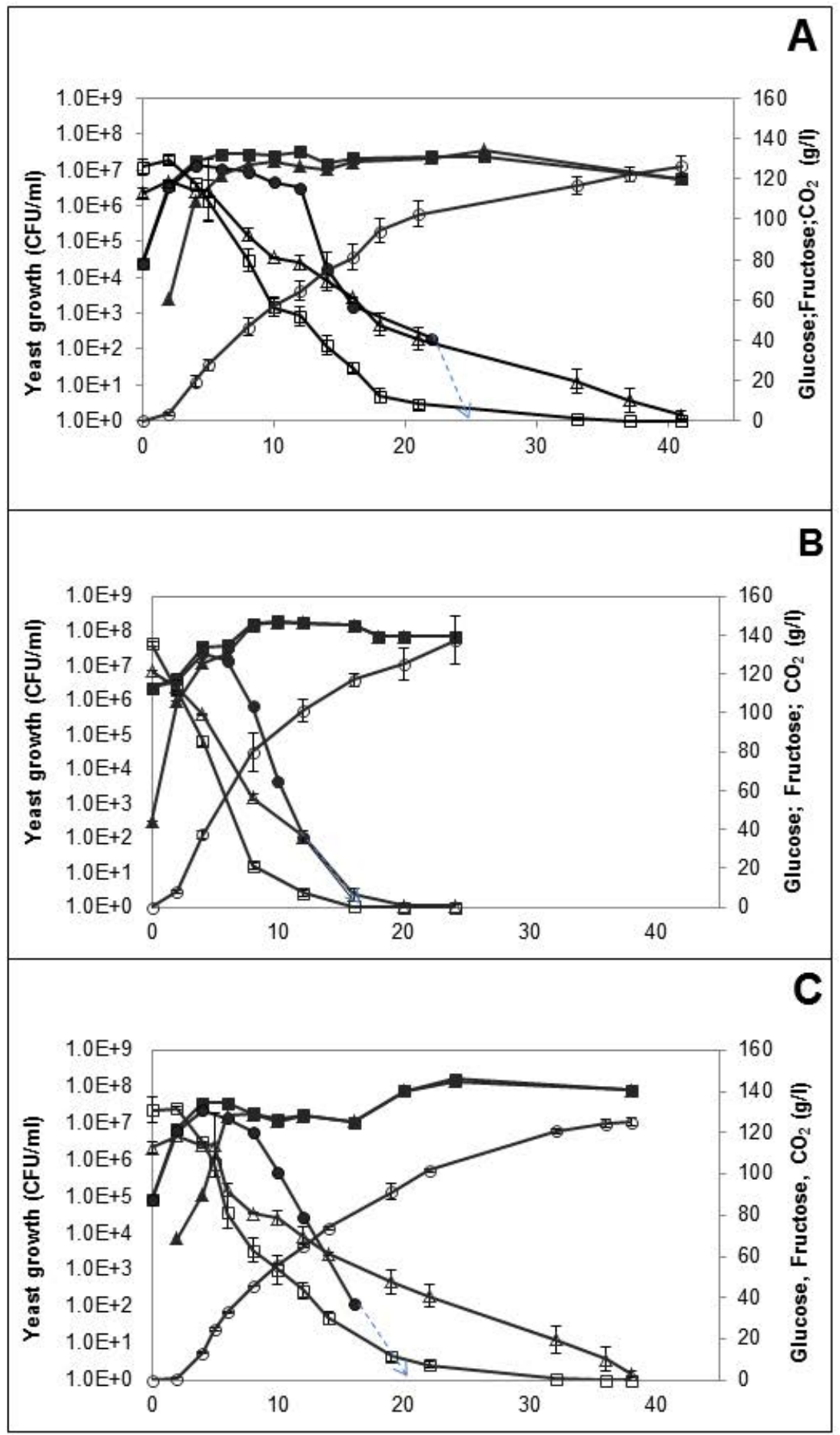

FIGURE 1

Fermentation kinetics demonstrating the yeast growth, $\mathrm{CO}_{2}$ evolution and sugar consumption during spontaneous fermentation of the three musts from the conventional (A), biodynamic (B) and integrated (C) vineyard. The dashed arrow shows that the yeast population was below detection. Error bars indicate standard deviations of the means of duplicate measurements of three biological repeats. 


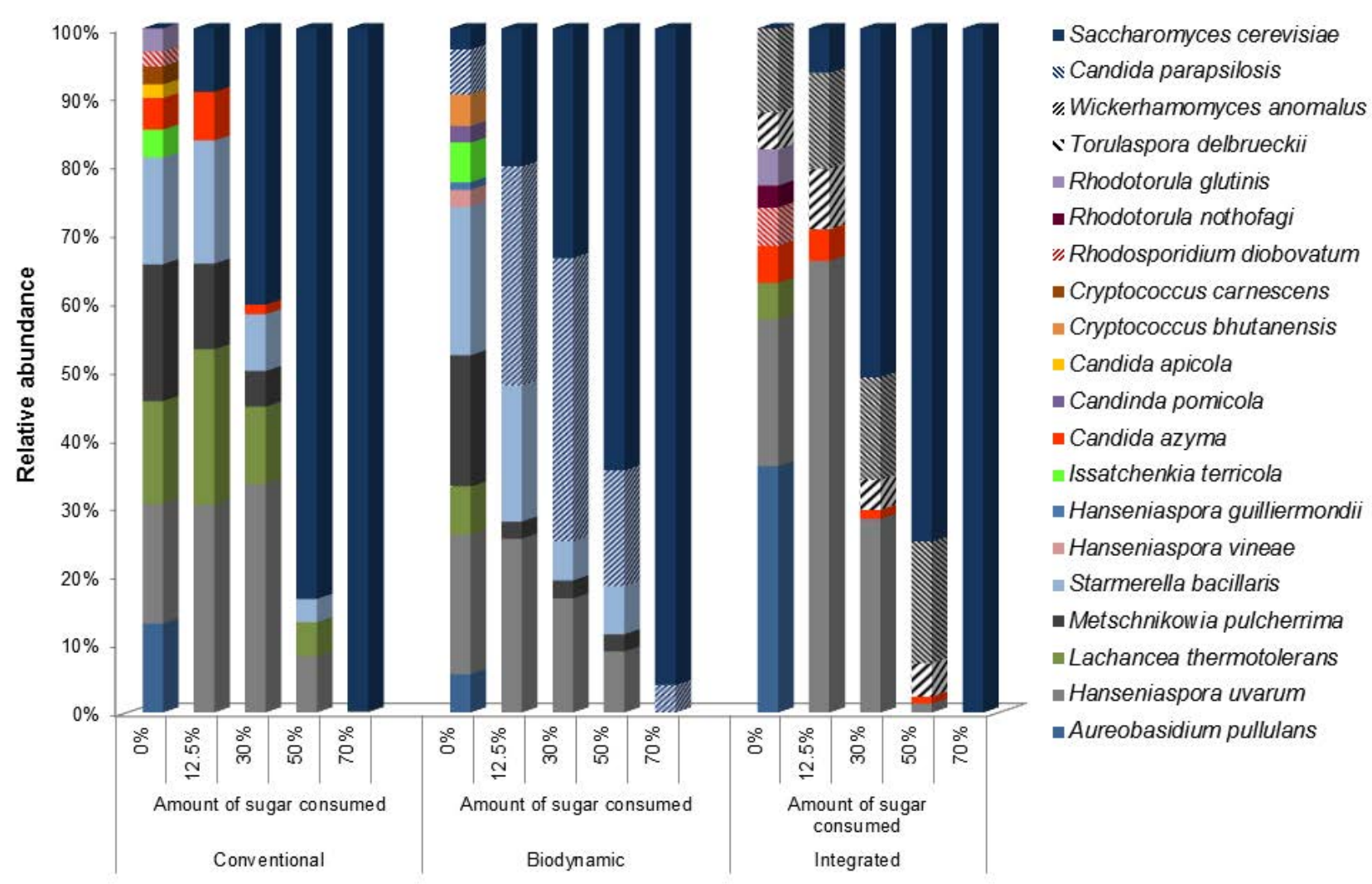

FIGURE 2

The occurrence and evolution of yeast species at different stages of spontaneous fermentation.

$S$. cerevisiae developed rapidly and was the dominant yeast by the middle of fermentation.

\section{DISCUSSION}

The current study evaluated the diversity of yeasts associated with Cabernet Sauvignon grapes obtained from biodynamic, conventional and integrated pest management farming systems. The dynamics of the yeast population during natural fermentation were monitored. The yeast population over two vintages (2012 and 2013) was $10^{4}$ to $10^{6} \mathrm{CFU} / \mathrm{mL}$, as typically found in grape must. The nonSaccharomyces species completely dominated the grape must yeast community, with $A$. pullulans, $H$. uvarum, $M$. pulcherrima and $L$. thermotolerans being common across the three vineyards. A. pullulans was previously shown to be the most dominant yeast species on the surface of undamaged grapes in the three vineyards, with other yeasts, such as Issatchenkia terricola, Cryptococcus carnescens, Rhodosporidium diobovatum, Rhodotorula nothofagi, Rhodotorula glutinis and Kazachstania aerobia, present only in lower concentrations (Setati et al., 2012). Members of the genus Kazachstania, which is closely related to Saccharomyces, are not commonly found in yeast isolates from grape must or wine. However, the first species to be described in this genus, Kazachstania viticola, was isolated from grapes. Subsequently, another species, Kazachstania hellenica, was isolated from fermenting must (Nisiotou \& Nychas, 2008), confirming the association of this genus with the vineyard and wine fermentation milieu. The impact that these yeasts have on wine quality remains to be elucidated. Although the isolation of these yeasts in the three vineyards seems inconsistent, it can be suggested that they are resident members of the yeast community in these vineyards and that their successful isolation depends on their level of abundance at the time of harvest. The fermentation data show that A. pullulans, Rhodotorula spp., Cryptococcus spp. and $R$. diobovatum are the first group of yeasts to decline when fermentation commences, probably due to their sensitivity to the prevailing anaerobic conditions. However, other studies have shown that some species of Rhodotorula, e.g. R. mucilaginosa, can persist throughout fermentation (Díaz et al., 2013). S. cerevisiae was either below detection or present only in low levels, as is evident in the biodynamic must sample, in which it accounted for 3 to $5 \%$ of the total yeast population. This representation is consistent with other studies, which have confirmed that this species is not dominant in the vineyard and usually occurs at approximately 10 to $100 \mathrm{CFU} / \mathrm{g}$ berries or less (Fleet, 2003).

Vintage variation in yeast diversity was evident in the current study. Indeed, in each must, whether from the same vineyard at different vintages or from different vineyards for a given vintage, different non-Saccharomyces yeasts persisted at higher levels for a significant part of the fermentation process. For instance, in the integrated vineyard, H. uvarum was the only species retrieved from the 2012 grape must, while nine different species were found in 2013. This could 
imply that the other species, although present on the grape surface, might have been present at significantly lower levels than $H$. uvarum. In contrast, $S$. bacillaris accounted for more than $20 \%$ of the population in the 2013 must from the biodynamic and conventional vineyards, while it was not detected in 2012. This yeast has most commonly been associated with botrytised grapes or high-sugar grape musts (Tofalo et al., 2012). Incidentally, the 2013 musts from the two vineyards contained higher sugar levels $(260 \mathrm{~g} / \mathrm{L}$ compared to 239 and $210 \mathrm{~g} / \mathrm{L}$ in the 2012 musts from the biodynamic and conventional vineyards, respectively). Therefore, the high levels of $S$. bacillaris could be a consequence of the presence of riper berries during harvesting and crushing. Overall, a higher representation of fermentative yeasts was evident in the 2013 musts from the three vineyards compared to the 2012 samples. The discrepancy in yeast diversity in consecutive years is not unusual and has been reported by other researchers, sometimes attributed to changes in climatic conditions, berry damage and berry ripeness (Combina et al., 2005; Díaz et al., 2013). In a recent study, Vigentini et al. (2015) used the Shanon Wiener index $\left(H^{\prime}\right)$ and multivariate data analysis to demonstrate that vintage rather than terroir had a more significant impact on yeast diversity associated with grapes. The data from the current study also show that the biodynamic vineyard consistently exhibited higher yeast species richness and diversity compared to the other farming practices. Although no studies have been done on the fermentation of grape must from biodynamic farms, previous studies have shown that grape must derived from organic vineyards tends to exhibit higher species and strain richness than that from conventional farms (Cordero-Bueso et al., 2011; Tello et al., 2011; Tofalo et al., 2011). These studies reported high biodiversity as reflected in higher $H^{\prime}$ values and low dominance $(D)$ in organic vineyards compared to conventional vineyards.

The fermentation dynamics in the 2013 fermentations of the three musts were very similar. However, the fermentation of the must from the biodynamic vineyard proceeded at a faster rate in comparison to the other two fermentations. The rapid rate of fermentation coincided with the high initial cell concentration $\left(10^{6} \mathrm{CFU} / \mathrm{mL}\right)$ and a higher percentage $(62 \%)$ of fermentative yeasts comprising six species, viz. H. uvarum, Hanseniaspora vineae, Hanseniaspora guilliermondii, $S$. bacillaris, $L$. thermotolerans and $S$. cerevisiae. The high fermentation tempo undoubtedly also was due to the rapid increase in $S$. cerevisiae. The must from the integrated vineyard, which contained approximately $45 \%$ fermentative yeasts and an initial cell concentration of $10^{5}$ $\mathrm{CFU} / \mathrm{mL}$, was second to finish fermentation, while the must from the conventional vineyard, with the lowest initial cell concentration $\left(10^{4} \mathrm{CFU} / \mathrm{mL}\right)$ and a lower percentage $(33 \%)$ of fermentative yeasts, took longer to ferment. This data confirm that community composition and cell concentration are important fermentation drivers, and that the percentage of fermentative yeasts compared to non-fermentative yeasts might be a good predictor of future fermentation performance. Similar observations have been made in other studies (Combina et al., 2005; Sun et al., 2009; CorderoBueso et al., 2011). The beginning of all three fermentations was dominated by weakly fermentative yeasts of the genera
Candida and Hanseniaspora at the level of 79.8\%, 90.7\% and $93.5 \%$ for the musts obtained from the biodynamic, conventional and integrated vineyards respectively. The species heterogeneity declined by the middle of fermentation, although different species were observed in the three fermentations. The must from the biodynamic vineyard displayed a high incidence of $C$. parapsilosis, and this yeast remained dominant throughout fermentation. C. parapsilosis was also previously shown to associate with the grapes from the biodynamic vineyard (Setati et al., 2012), and although it is not a common wine yeast, its presence in grape must and persistence during wine fermentation has been reported (Zott et al., 2008; Clavijo et al., 2010). This yeast has weaker glycolytic enzyme activity compared to $S$. cerevisiae and does not outcompete $S$. cerevisiae during wine fermentation. The fermentation kinetics in the must from the biodynamic vineyard also show that fructose consumption was relatively fast. This must had the highest initial level of $S$. bacillaris, which is fructophilic and persisted until $50 \%$ of the sugar was consumed. The data suggest that the two Candida species contributed positively to the fermentation kinetics, as their persistence did not retard the fermentation rate.

In the must from the integrated vineyard, Wickerhamomyces anomalus was the second dominant yeast after $H$. uvarum. This yeast has previously been associated with grape must and was shown to persist until the end of fermentation (Renouf et al., 2007; Díaz et al., 2013). In addition, some strains of this yeast can tolerate up to $12.5 \%$ $(\mathrm{v} / \mathrm{v})$ ethanol and are known to produce killer toxins (Walker, 2011; Sabel et al., 2014). This could allow W. anomalus to compete against the other yeasts in the same environment. However, this would depend on whether the killer toxins are active under wine fermentation conditions. It was observed in the current study that the cell concentration of $W$. anomalus only increased marginally throughout fermentation, suggesting that its growth is severely hampered by the lack of oxygen. This yeast generally shows low growth rates $\left(0.056 \mathrm{~h}^{-1}\right)$ and biomass yields $(0.11 \mathrm{~g} / \mathrm{g}$ glucose $)$ under anaerobic conditions (Walker, 2011). Therefore, the slight population increase was probably induced by the brief introduction of oxygen during sampling. Interestingly, $L$. thermotolerans and T. delbrueckii are good fermentative yeasts and some strains are now available commercially as starter cultures. Both species, however, did not persist until the end of fermentation. The decline of these yeasts during wine fermentation has been attributed to their high biosynthetic oxygen requirement (Hansen et al., 2001; Hanl et al., 2005). However, L. thermotolerans, which was present in all three fermentations, behaved differently across the fermentations, suggesting that yeast-yeast interactions as well as the initial cell density play a significant role in its dynamics. For instance, in the conventional must, in which the initial cell density was higher, L. thermotolerans survived until the middle of fermentation, whereas in the other two fermentations it died off in the early fermentation stage. This indicates that a high initial cell concentration is crucial to allow this yeast to establish itself during fermentation. It also is possible that the growth of this yeast in the integrated and biodynamic must fermentations may have been inhibited by competing yeasts. For instance, in the biodynamic must, 
rapid nutrient utilisation by $C$. parapsilosis and $S$. cerevisiae, coupled with oxygen limitation and ethanol build-up, could be responsible for the suppression of $L$. thermotolerans. Similarly, T. delbrueckii, which has been reported to have a strong fermentative activity and to tolerate up to $10 \%(\mathrm{v} / \mathrm{v})$ ethanol (Cordero-Bueso et al., 2011; Xufre et al., 2006), could not persist until the end of fermentation and only maintained low levels until the middle of fermentation in the must from the integrated vineyard.

Overall, non-Saccharomyces yeast species with known potential to affect the sensory quality of wine persisted in high numbers for a significant part of the fermentation. However, these species were different for each of the musts that were studied here, indicating the highly variable nature of natural fermentation. Importantly, and in all cases, persisting species were already well represented in the initial must, indicating that knowledge of the initial mycobiome would be an important management tool for winemakers in order to enhance or suppress existing dominant species. In addition, the data support the idea that a high biodiversity, including a mix of fermentative and non-fermentative glucophilic and fructophilic yeasts is beneficial to overall fermentative activity. The data also show that the ratio of fermentative to oxidative yeasts in the initial must is a crucial factor, since a low initial concentration of fermentative yeasts leads to a slow fermentation tempo. The dominance and persistence of non-Saccharomyces yeast species depended strongly on the initial cell concentration of a given species, the sensitivity to oxygen limitation and the ethanol concentration. Furthermore, the ability of $S$. cerevisiae to establish itself and dominate the fermentation appears not to be affected fundamentally by a low initial cell concentration and the presence of a diverse range of competing yeast consortia.

\section{LITERATURE CITED}

Barata, A., Malfeito-Ferreira, M. \& Loureiro, V., 2012a. The microbial ecology of wine grape berries. Int. J. Food Microbiol. 153, 243-259.

Barata, A., Santos, S.C., Malfeito-Ferreira, M. \& Loureiro, V., 2012b. New insights into the ecological interaction between grape berry microorganisms and Drosophila flies during the development of sour rot. Microb. Ecol. 64, 416-430.

Bezerra-Bussoli, C., Baffi, M.A., Gomes, E. \& Da-Silva, R., 2013. Yeast diversity isolated from grape musts during spontaneous fermentation from a Brazilian winery. Curr. Microbiol. 67, 356-361.

Clavijo, A., Calderón, I.L. \& Paneque, P., 2010. Diversity of Saccharomyces and non-Saccharomyces yeasts in three red grape varieties cultured in the Serranía de Ronda (Spain) vine-growing region. Int. J. Food Microbiol. 143, 241-245.

Combina, M., Elía, A., Mercado, L., Catania, C., Ganga, A. \& Martinez, C., 2005. Dynamics of indigenous yeast populations during spontaneous fermentation of wines from Mendoza, Argentina. Int. J. Food Microbiol. 99, 237-243.

Cordero-Bueso, G., Arroyo, T., Serrano, A., Tello, J., Aporta, I., Vélez, M.D. \& Valero, E., 2011. Influence of the farming system and vine variety on yeast communities associated with grape berries. Int. J. Food Microbiol. $145,132-139$.

Díaz, C., Molina, A.M., Nähring, J. \& Fischer, R., 2013. Characterization and dynamic behavior of wild yeast during spontaneous wine fermentation in steel tanks and amphorae. BioMed. Res. Int. Article ID 540465.
Di Maro, E., Ercolini, D. \& Coppola, S., 2007. Yeast dynamics during spontaneous wine fermentation of the Catalanesca grape. Int. J. Food Microbiol. 117, 201-210.

Duarte, F.L., Pimentel, N.H., Teixeira, A. \& Fonseca, À., 2012. Saccharomyces bacillaris is not a synonym of Candida stellata: Reinstatement as Starmerella bacillaris comb. nov. Antonie van Leeuwenhoek 102, 653-658.

Esteve-Zarzoso, B., Belloch, C., Uruburu, F. \& Querol, A., 1999. Identification of yeasts by RFLP analysis of the 5.8S rRNA gene and the two ribosomal internal transcribed spacers. Int. J. Syst. Bacteriol. 49, 329-337.

Fleet, G.H. 2003. Yeast interactions and wine flavour. Int. J. Food Microbiol. $86,11-22$.

Hanl, L., Sommer, P. \& Arneborg, N., 2005. The effect of decreasing oxygen feed rates on growth and metabolism of Torulaspora delbrueckii. Appl. Microbiol. Biotechnol. 67, 113-118.

Hansen, E.H., Nissen, P., Sommer, P., Nielsen, J.C. \& Arneborg, N., 2001. The effect of oxygen on the survival of non-Saccharomyces yeasts during mixed culture fermentations of grape juice with Saccharomyces cerevisiae. J. Appl. Microbiol. 91, 541-547.

Hoffman, C.S., 2003. Rapid isolation of yeast chromosomal DNA. In: Ausubel, F.M., Brent, R., Kingston, R.E., Moore, D.D., Seidman, J.G., Smith, J.A. \& Struhl, K., (eds). Current protocols in molecular biology. John Wiley \& Sons Inc., Place of publication? Supplement 39.

Milanović, V., Comitini, F. \& Ciani, M., 2013. Grape berry yeast communities: Influence of fungicide treatments. Int. J. Food Microbiol. $161,240-246$.

Mortimer, R. \& Polsinelli, M., 1999. On the origins of wine yeast. Res. Microbiol. 150, 199-204.

Nisiotou, A.A. \& Nychas, G.-H.E., 2008 Kazachstania hellenica sp. nov., a novel ascomycetous yeast from a Botrytis-affected grape must fermentation. Int. J. Syst. Evol. Microbiol. 58, 1263-1267.

Povhe Jemec, K., Cadez, N., Zagorc, T., Bubic, V., Zupec, A. \& Raspor, P., 2001. Yeast population dynamics in five spontaneous fermentations of Malvasia must. Food Microbiol. 18, 247-259.

Prakitchaiwattana, C.J., Fleet, G.H. \& Heard, G.M., 2004. Application and evaluation of denaturing gradient gel electrophoresis to analyse the yeast ecology of wine grapes. FEMS Yeast Res. 4, 856-877.

Rementeria, A., Rodriguez, J.A., Cadaval, A., Amenabar, R., Muguruza, J.R., Hernando, F.L. \& Sevilla, M.J., 2003. Yeast associated with spontaneous fermentations of white wines from the "Txakoli de Bizkaia" region (Basque Country, North Spain). Int. J. Food Microbiol. 86, 201-207.

Renouf, V., Claisse, O. \& Lonvaud-Funel, A., 2005. Understanding the microbial ecosystem on the grape berry surface through numeration and identification of yeast and bacteria. Aust. J. Grape Wine Res. 11, 316-327.

Renouf, V., Claisse, O. \& Lonvaud-Funel, A., 2007. Inventory and monitoring of wine microbial consortia. Appl. Microbiol. Biot. 75, 149-164.

Sabel, A., Martens, S., Petri, A., König, H. \& Claus, H., 2014. Wickerhamomyces anomalus AS1: A new strain with potential to improve wine aroma. Ann. Microbiol. 64, 483-491.

Setati, M.E., Jacobson, D., Andong, U.C. \& Bauer, F., 2012. The vineyard yeast microbiome, a mixed model microbial map. PLoS ONE 7(12), e52609. doi:10.1371/journal.pone.0052609

Stefanini, I., Dapporto, L., Legras, J.-L., Calabretta, A., Di Paola, M., De Filippo, C., Viola, R., Capretti, P., Polsinelli, M. \& Turillazzi, S., 2012. Role of social wasps in Saccharomyces cerevisiae ecology and evolution. PNAS Early edition. 1-6. Available at: www.pnas.org/cgi/doi/10.1073/ pnas. 1208362109 
Sturm, J., Grossmann, M. \& Schnell, S., 2006. Influence of grape treatment on the wine yeast populations isolated from spontaneous fermentations. J. Appl. Microbiol. 101, 1241-1248.

Sun, H., Ma, H., Hao, M., Pretorius, I.S. \& Chen, S., 2009. Identification of yeast population dynamics of spontaneous fermentation in Beijing wine region, China. Ann. Microbiol. 59, 69-76.

Tello, J., Cordero-Bueso, G., Aporta, I., Cabellos, J.M. \& Arroyo, T., 2011. Genetic diversity in commercial wineries: Effects of the farming system and vinification management on wine yeasts. J. Appl. Microbiol. 112, 302-315.

Tofalo, R., Chaves-López, C., Di Fabio, F., Schirone, M., Felis, G.E., Torriani, S., Paparella, A. \& Suzzi, G., 2009. Molecular identification and osmotolerant profile of wine yeasts that ferment a high sugar grape must. Int. J. Food Microbiol. 130, 179-187.

Tofalo, R., Schirone, M., Telera, G.C., Manetta, A.C., Corsetti, A. \& Suzzi, G., 2011. Influence of organic viticulture on non-Saccharomyces yeast populations. Ann. Microbiol. 61, 57-66.
Tristezza, M., Vetrano, C., Bleve, G., Spano G., Capozzi, V., Logrieco, A., Mita, G. \& Grieco, F., 2013. Biosafety aspects of yeast strains characterized from vineyards and spontaneous fermentations in the Apulia region, Italy. Food Microbiol. 36, 335-342.

Vigentini, I., De Lorenzis, G., Fabrizio, V., Valdetara, F., Faccincani, M., Panont, C.A., Picozzi, C., Imazio, S., Failla, O. \& Foschino, R., 2015. The vintage effect overcomes the terroir effect: A three years survey on the wine yeast biodiversity in Franciacorta and Oltrepò Pavese, two Northern Italian vine-growing areas. Microbiol. 161(2), 362-373. doi:10.1099/mic.0.000004

Walker, G.M., 2011. Pichia anomala: Cell physiology and biotechnology relative to other yeasts. Antonie van Leeuwenhoek 99, 25-34.

Xufre, A., Albergaria, H., Inácio, J., Spencer-Martins, I. \& Girio, F., 2006. Application of fluorescence in situ hybridisation (FISH) to analysis of yeast population dynamics in winery and laboratory grape must fermentations. Int. J. Food Microbiol. 108, 376-384.

Zott, K., Miot-Sertier, C., Claisse, O., Lonvaud-Funel, A. \& MasneufPomarede, I., 2008. Dynamics and diversity of non-Saccharomyces yeasts during the early stages in winemaking. Int. J. Food Microbiol. 125, 197-203. 